\title{
Effects of Penetration Enhancers for Transdermal Drug Delivery System and Methods Used for Them
}

\author{
Guangyu Xu ${ }^{1, \text { a }}$, Bin Zhao ${ }^{2,3, \text { a } \text {, Guanghong Wang }}{ }^{1, c}$ and Manli Wang ${ }^{1, d^{*}}$ \\ ${ }^{1}$ College of Pharmacy, Beihua University, 3999 East Binjiang Road, Fengman District, Jilin, Jilin, \\ 132013, People's Republic of China \\ ${ }^{2}$ Affiliated hospital of Beihua University, 12 Jiefang road, Chuanying District, Jilin, Jilin, 132013, \\ People's Republic of China \\ ${ }^{3}$ General Hospital of CNPC in Jilin, 52 Zunyi Road, Longtan District, Jilin, Jilin, 132013, People's \\ Republic of China
}
a25796845@qq.com, ${ }^{b} 54218098 @ q q . c o m,{ }^{c} 578311398 @ q q . c o m$, dbhwangml@163.com
* Corresponding author: Manli Wang, Email: bhwangml@163.com

Keywords: Transdermal penetration enhancers; Traditional Chinese medicine; Chemical synthesis penetration enhancers.

Abstract. Among methods used for transdermal penetration enhancement of drugs, the application of transdermal penetration enhancers is a method that has been most studied currently to promote the transdermal absorption of drugs, and also the most convenient method. Transdermal penetration enhancers (penetration enhancers) are defined as substances that you can accelerate the speed of the drug to penetrate the skin without severe irritation and damage to the skin. In this paper, the main mechanism of action and application methods of penetration enhancers for transdermal drug delivery system are comprehensively reviewed, which is expected to provide some helpful information for the relevant researchers. With the deepening of research on methods for the transdermal drug delivery system, more and more methods for the transdermal penetration enhancement have been learned. However, to look for safe and effective penetration enhancers for transdermal drug delivery system is still a research priority. Some new techniques for the detection and analysis are still required for elucidating structure-activity relationships and mechanism of action of the penetration enhancers, such as micro-dialysis and other methods. In addition, the synergistic effect of physical penetration enhancement method combined with chemical methods is also an important direction for the future research.

\section{Introduction}

Transdermal drug delivery system (TDDS) is a preparation used for the treatment or prevention of diseases, in which a transdermal patch is used to administer a drug and the drug is absorbed through the skin into the bloodstream to reach the effective blood concentration. However, due to the barrier function of the skin, as well as the physical and chemical properties of drugs themselves and other reasons, many transdermal drug absorption rates of many drugs are very low, so that the drugs absorbed through the skin is difficult to achieve an effective therapeutic concentration. Therefore, the addition of a certain amount of penetration enhancers for the transdermal absorption to a preparation of the transdermal drug delivery system has become a key link to overcome the barrier function of skin and promote the transdermal absorption of drugs.

Among methods for increasing the transdermal permeability of drugs, the application of penetration enhancers for transdermal absorption is a method that has been most studied for promoting the transdermal absorption of drugs, and also a method that is the most convenient currently[1]. Transdermal absorption enhancers (called for short, penetration enhancers) are referred to substances that can accelerate the transdermal speed of drugs but not cause a severe irritation and damage to the skin. In this paper, the main mechanism of action and application methods of penetration enhancers for transdermal drug delivery system are comprehensively reviewed, which is expected to provide some helpful information for the relevant researchers. 


\section{Mechanisms for Enhancing the Transdermal Absorption of Drugs}

Based on the structural characteristics of the stratum corneum and the continuity of lipid barrier, the interaction of penetration enhancers and the stratum corneum lipids is crucial for the effectiveness of penetration enhancers. The site of action of most transdermal penetration enhancers is the lipid of stratum corneum and its liquidity can be increased by breaking the ordered arrangement of stratum corneum lipid. After the distribution into the stratum corneum of skin, penetration enhancers interact with components in the stratum corneum to reduce the barrier function of skin to promote the transdermal absorption of drugs. In general, penetration enhancers exert their transdermal penetration enhancement through the following mechanism [2,3]: 1) they act on the intercellular lipid, especially the ceramide, to break the ordering in the structure of the stratum corneum; 2) they act on proteins to change the conformation of proteins, such as keratin and desmoplakin in keratinocytes, thereby reducing the cohesion of the stratum corneum.

\section{Methods for Enhancing the Transdermal Absorption of Drugs}

There are three primary methods for ransdermal penetration enhancers to promote the absorption of drugs. The first method is a chemical one that is mostly used currently, in which the absorption of drugs is promoted mainly by chemical agents, primarily including transdermal penetration enhancers of traditional Chinese medicine and chemically synthesized transdermal penetration enhancers; the second method is a biological one, in which some biological macromolecular penetration enhancers are used, with a high transdermal penetration efficiency, less side effect, a strong specificity, but higher technical requirement and high cost; the third method is a physical one mainly using ion method and ultrasonic method that are limitedly applied.

\section{Chemical methods}

The chemical method is one that the transdermal absorption of drugs is enhanced by penetration enhancers. It is the main method currently used for promoting the transdermal absorption of drugs. There are any different types of penetration enhancers, which can be mainly divided into the following two categories:

Transdermal penetration enhancers of traditional Chinese medicine. Transdermal penetration enhancers of traditional Chinese medicine have shown some advantages in rapid onset, good effect, fewer side effects, low cost, etc., with a broad prospect for the development. In the research field of transdermal penetration enhancers of traditional Chinese medicine, the most studies focus on borneol, mint, and volatile oils of a variety of traditional Chinese medicine and their extracts, and the application of transdermal penetration enhancers of traditional Chinese medicine is both simple and in combination with each other. (1)Menthol: Menthol is also known as menthol crystal, one of cyclohexane-like monoterpenes derivatives and present in peppermint oil. In recent years, a composite penetration enhancer containing menthol discovered by scholars can significantly improve the penetration enhancement[4]. (2) Volatile oils: there are many Chinese medicines with the transdermal enhancement, such as cinnamon volatile oil, rhizoma zingiberis volatile oil, rhizoma galangae volatile oil, atratylis oil and patchouli oil. (3) Borneol: Borneol has functions such as resuscitation, clearing heat and analgesia, and is rarely used alone and often used as "a primer and adjuvant drug". Yuan zhizhao, et al experimentally confirmed that the amount of transdermal penetration of fluorouracil and dexamethasone in the vitiligo cream ointment containing $0.3 \%$ or $0.5 \%$ borneol was significantly increased. Some scholars have found that the penetration enhancement of borneol on the drugs is produced mainly in the stratum corneum. It has been also found that the combined use of borneol and menthol can enhance the transdermal penetration.[5]

Chemically synthesized penetration enhancers. The effect of penetration enhancement produced by chemically synthesized penetration enhancers is better and strongly targeted. 
Chemically synthesized penetration enhancers that are widely used are mainly as follows: (1) Organic acids and their esters: They can significantly increase the transdermal absorption of levodopa, dopamine hydrochloride and isoproterenol hydrochloride. The penetration enhancement of amino acid esters, such as $\mathrm{N}, \mathrm{N}$ - dimethyl amino dodecyl acetate, on indomethacin is similar to that of azone, with a faster onset and smaller stimulation to the skin than that of azone. (2) Pyrrolidones: For example, 1-lauroyl-2-pyrrolidone can significantly increase the transdermal permeability of aminopyrines.

\section{Biological methods}

Enzymes. Researchers have confirmed that enzymes can significantly affect or adjust the transdermal absorption of drugs. Phosphatase $\mathrm{C}$, triglyceride hydrolase and phosphatases $\mathrm{A}_{2}$ can increase the transdermal rate of mannitol by 4.4, 3.5 and 3.3 folds, respectively. In addition, phosphatase $\mathrm{C}$ can also increase the steady-state transdermal rate of benzoic acid and testosterone by 5.7 and 1.7 times, respectively[6].

Cyclodextrins. The clathration of a variety of active molecules by cyclodextrin can improve the transdermal absorption of drugs. However, some scholars found that the transdermal penetration enhancement of cyclodextrin on polar or lipid-soluble drugs did not rise but decreased when they studied the penetration enhancement of cyclodextrins on para-toluene and terpenes[7].

Synthetic lipid inhibitors. Some researchers[8] disturbed the skin barrier layer with acetone and dimethyl sulfoxide, and then further prevented the recovery of functions of the skin barrier layer with fatty acid synthesis inhibitors, cholesterol synthesis inhibitor or cholesteryl sulfate, thereby further increasing the absorption of lidocaine and making the absorption of lidocaine absorbed more in combination of them with lidocaine. However, the physicochemical effect of this kind of inhibitors alone can not change the absorption of drugs through an intact skin. The main reason of transdermal penetration enhancement is that these substances can alter the metabolic balance and thermodynamic activity of the permeation barrier. The combination of this metabolic method with chemical penetration enhancers may provide a new method for the transdermal absorption of drugs.

\section{Physical methods}

Ion method. Ion method is a most commonly used and also most effectively physical method in the transdermal drug delivery, in which the transdermal absorption of drugs into the body through the skin is driven by a physiologically tolerated current. This method is particularly suitable for protein and polypeptide drugs that can be degraded by enzymes in the gastrointestinal tract and affected by the hepatic first-pass effect, such as insulin, thyrotropin-releasing hormone, vasopressin, etc. Studies have found that only a constant direct current has the penetration enhancement, and pulsed direct current and alternating current have no effect almost[9].

Ultrasonic method. Ultrasonic method is a method to utilize piezoelectric crystals to generate ultrasonic energy after they receive an alternating current to generate a vibration to make the absorbent medium come into being a mechanical disorder or alter the lipid structure of stratum corneum, thereby promoting the absorption of drugs. The most important feature of the method is that the absorption of drugs can be increased within a short time. Physicochemical properties of drugs have a significant impact on the penetration enhancement of ultrasound, of which in addition to the molecular weight of drugs, the lipophilicity of drugs is also an important factor. Ueda [10] studied the penetration enhancement of ultrasound on different drugs and found that the transdermal penetration enhancement of ultrasound on water-soluble drugs, especially protein and peptide drugs, was particularly significantly, but it had no the penetration enhancement on lipophilic drugs almost.

With the deepening of research on methods for the transdermal drug delivery system, more and more methods for the transdermal penetration enhancement have been learned. However, to look for safe and effective penetration enhancers for transdermal drug delivery system is still a research priority. Some new techniques for the detection and analysis are still required for elucidating 
structure-activity relationships and mechanism of action of the penetration enhancers, such as microdialysis and other methods. In addition, the synergistic effect of physical penetration enhancement method combined with chemical methods is also an important direction for the future research.

\section{Acknowledgements}

This work was supported by Science and technology development project of Jilin Province (Grant No. 20140520048JH) and Scientific and technological research project during the 12th Five-Year Plan Period of Jilin Provincial Education Department (Grant No. 214(2014)).

\section{References}

[1] B. Barry, Breaching the skin's barrier to drugs, Nat Biotechnol. 22 (2004) 165-167.

[2] A. Williams and B. Barry, Terpenes and the lipid-protein-partitioning theory of skin penetration enhancement, Pha20140520048rmaceutical Research, 8 (1991) 17-24.

[3] A. Williams and B. Barry, Penetration enhancers, Advanced Drug Delivery Reviews, 56 (2004) 603-618.

[4] Y. Lan, J. Wang, H. Li, Y. Zhang, Y. Chen, B. Zhao, Q. Wu, Effect of menthone and related compounds on skin permeation of drugs with different lipophilicity and molecular organization of stratum corneum lipids, Pharm Dev Technol. 16 (2015) 1-10.

[5] L. Shan, G. Jin, P. Wang, S. Liu, Research of Traditional Chinese Medicine Transdermal Enhancers, Journal of Liaoning University of TCM 15 (2013) 112-114.

[6] S. Patil, P. Singh, C. Szolar-Platzer, H. Maibach, Epidermal enzymes as penetration enhancers in transdermal drug delivery? J Pharm Sci. 85(1996) 249-252.

[7] A. Williams, S. Shatri, B. Barry, Transdermal permeation modulation by cyclodextrins: a mechanistic study, Pharm Dev Technol. 3(1998) 283-296.

[8] F. Foglia, S. Rogers, J. Webster, F. Akeroyd, Neutron Scattering Studies of the Effects of Formulating Amphotericin B with Cholesteryl Sulfate on the Drug's Interactions with Phospholipid and Phospholipid-Sterol Membranes, Langmuir. 31(2015) 8042-8051.

[9] T. Kezutyte, N. Desbenoit, A. Brunelle, V. Briedis, Studying the penetration of fatty acids into human skin by ex vivo TOF-SIMS imaging, Biointerphases. 8 (2013) 3-5.

[10] H. Ueda, M. Mutoh, T. Seki, D. Kobayashi, Y. Morimoto, Acoustic cavitation as an enhancing mechanism of low-frequency sonophoresis for transdermal drug delivery, Biol Pharm Bull. 32 (2009) 916-920. 